\title{
Systems of Spatial Reference in Human Memory
}

\author{
Amy L. Shelton
}

Stanford University

and

\author{
Timothy P. McNamara
}

Vanderbilt University

\begin{abstract}
Seven experiments examined the spatial reference systems used in memory to represent the locations of objects in the environment. Participants learned the locations of common objects in a room and then made judgments of relative direction using their memories of the layout (e.g., "Imagine you are standing at the shoe, facing the lamp; point to the clock"). The experiments manipulated the number of views that observers were allowed to experience, the presence or absence of local and global reference systems (e.g., a rectangular mat on which objects were placed and the walls of the room, respectively), and the congruence of local and global reference systems. Judgments of relative direction were more accurate for imagined headings parallel to study views than for imagined headings parallel to novel views, even with up to three study views. However, study views misaligned with salient reference systems in the environment were not strongly represented if they were experienced in the context of aligned views. Novel views aligned with a local reference system were, under certain conditions, easier to imagine than were novel views misaligned with the local reference system. We propose that learning and remembering the spatial structure of the surrounding environment involves interpreting the layout in terms of a spatial reference system. This reference system is imposed on the environment but defined by egocentric experience. ๑ 2001 Elsevier Science
\end{abstract}

Spatial reference systems are essential for the specification of location and orientation in space. The location of Nashville, for example, can be specified by describing its position with respect to the boundaries of the state (e.g., Nashville is in the middle of Tennessee), by providing coordinates of latitude

The authors thank Jeffrey Schall, Kyle Cave, Jeffery Franks, and Ted Payne for various contributions to the research. We also thank the reviewers for comments on earlier drafts. This research was supported in part by grants from the National Science Foundation (SBR9222002) and the National Institutes of Mental Health (R01-MH57868 and F32-MH12638).

Address correspondence and reprint requests to Amy L. Shelton, Department of Psychology, Jordan Hall, Bldg 420, Stanford University, Stanford, CA 94305. 
and longitude on the surface of the Earth (e.g., Nashville is located at $36^{\circ} \mathrm{N} 09: 57$ and $\left.86^{\circ} \mathrm{W} 47: 04\right)$, or by describing its position relative to an observer (e.g., Nashville is 2300 miles east of where the first author was sitting when she wrote this paragraph). In each case, a spatial reference system is used-and indeed required-to identify location. Humans store spatial information in memory about many familiar environments, and just as spatial reference systems are required to specify the locations of objects in physical space, so too spatial reference systems must be used by the human memory system to represent the locations of objects in the environment. The principal goal of the experiments reported in this article was to determine the relative importance of, and possible interactions among, the spatial reference systems defined by the observer and by the external world in forming mental representations of the environment.

There has been an enormous amount of research in psychology and allied disciplines examining frames of reference in perception, attention, memory, and language. In the psychological literature, a frame of reference is commonly understood to establish orthogonal axes in two- or three-dimensional space (e.g., Carlson-Radvansky \& Irwin, 1993). A spatial reference system, as we use the term (and as it has been used by others, in particular, the early Gestalt psychologists; see e.g., Rock, 1992), is a more general concept and includes orthogonal axes as a special case. By this definition, a spatial reference system is a relational system consisting of located objects, reference objects, and the spatial relations that may obtain between them. The reference objects may be any objects whose positions are known or assumed as a standard and include the observer; landmarks; coordinate axes; the planes defined by the walls, floor, and ceiling of a room; and so forth (e.g., Talmy, 1983). Returning to the introductory examples, the first locates Nashville by specifying nonmetric spatial relations between Nashville and the state borders of Tennessee, which do not form orthogonal axes.

Although there are many ways to classify spatial reference systems (e.g., Levinson, 1996), an extremely useful one, for the purposes of understanding human spatial cognition, draws a distinction between egocentric and environmental reference systems. Egocentric reference systems specify location and orientation with respect to the observer (e.g., body-centered coordinates). Environmental reference systems define spatial relations with respect to elements of the environment, such as the perceived direction of gravity; landmarks; or the floor, ceiling, and walls of a room. The relative importance of egocentric and environmental reference systems in attention and perception has been explored in many studies (e.g., Attneave \& Reid, 1968; CarlsonRadvansky \& Irwin, 1993; Friedman \& Hall, 1996; Hinton, 1979; McMullen \& Jolicoeur, 1990; Palmer, 1989; Pani \& Dupree, 1994; Rock, 1973). As is shown below, however, very little is known about the relative importance of such spatial reference systems in representing navigable space in long-term memory. 


\section{Foundational Assumptions}

Before looking more closely at research on the spatial reference systems used in memory, we state explicitly the assumptions that underpin our interpretations of our own findings as well as the findings of other researchers. In all of the experiments reported in this article, participants first learned the locations of objects in a room from one or more viewpoints and then made judgments of relative direction using their memories of the layouts (e.g., "Imagine you are standing at the book, facing the wood; point to the clock"). Performance in this task was used to assess which views of the layouts were more or less accessible. We assume that spatial relations that are explicitly specified with respect to a particular spatial reference system can be retrieved from memory, whereas spatial relations that are not explicitly specified in terms of that spatial reference system must be inferred. These inferential processes produce measurable costs in terms of latency and error. Judgments based on retrieved spatial relations will therefore be faster and more accurate than judgments based on inferred spatial relations. Hence, we can use performance in judgments of relative direction as an index of the extent to which spatial relations were inferred, with poorer performance corresponding to a greater dependence on inferred, as opposed to retrieved, spatial relations.

The fact that people may represent spatial layouts in terms of egocentric reference systems does not imply that people cannot imagine nonegocentric perspectives. Indeed, one does not need to conduct an experiment to know that people can imagine, recognize, and talk about nonegocentric viewpoints. The point is that these nonegocentric judgments produce measurable costs; mental computation is involved. As discussed in more detail below, these costs are well documented in the spatial memory literature; they also occur, however, in lower level perceptual and attentional processes. For example, in Logan's (1995, Experiments 8 and 9) investigations of visual attention, participants had to identify the color of the stimulus appearing above, below, to the right of, or to the left of a cue. The direction was given prior to the stimulus array. Even in these relatively simple perceptual judgments, there was a cost of approximately $20 \%$ to adopting a nonegocentric/nonenvironmental frame of reference, in which the top of a figure was defined to be at $90^{\circ}, 180^{\circ}$, or $270^{\circ}$, relative to an egocentric/environmental frame of reference, in which top was at $0^{\circ}$. We suggest that this cost reflects the need to infer spatial relations that are not explicitly represented in perception or in memory.

\section{Egocentric Reference Systems}

The role of egocentric reference systems in spatial memory is, at this point, well documented (e.g., Diwadkar \& McNamara, 1997; Presson \& Montello, 1994; Rieser, 1989; Rieser, Guth, \& Hill, 1986; Roskos-Ewoldsen, McNamara, Shelton, \& Carr, 1998; Shelton \& McNamara, 1997a, 1997b; Sholl \& 


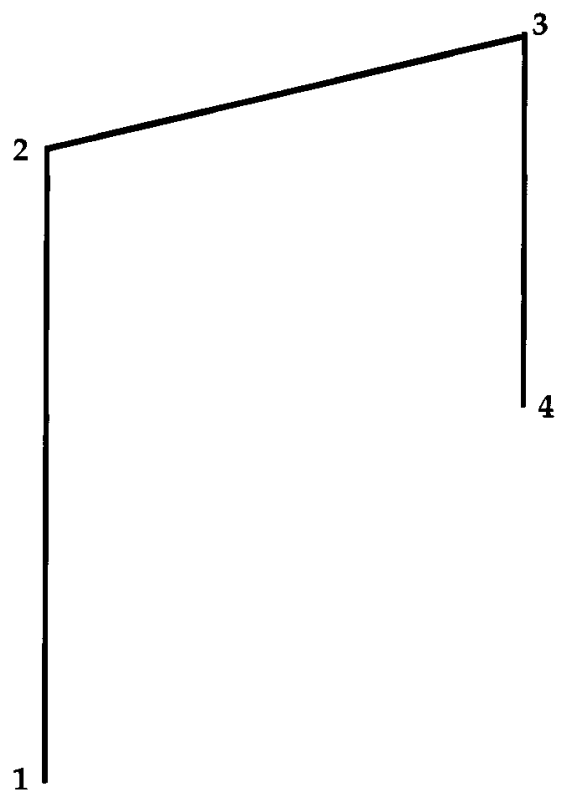

FIG. 1. Four-point path. Adapted from Roskos-Ewoldsen et al. (1998).

Nolin, 1997). For example, Roskos-Ewoldsen et al. (1998) had participants learn four-point paths (see Fig. 1) or displays of common objects (see Fig. 2) from a single viewpoint and then make judgments of relative direction using their memories of the layouts. The results showed that people were faster and more accurate imagining a perspective oriented with the position at study (e.g., Point to 3 as if standing at 1 facing 2 ) than imagining a perspective that differed from the one at study (e.g., Point to 1 as if standing at 3 facing 4). Rieser (1989) found similar results for circular displays of objects.

These and similar findings indicate that room-sized and smaller spatial layouts are mentally represented in terms of orientation-dependent reference systems. These reference systems specify spatial structure with respect to the orientation of the observer; examples include retinotopic coordinates and head-centered and body-centered reference systems. The aspect shared by all of these spatial reference systems is that the location of an object, object part, or feature cannot be described without making reference to the orientation of the observer in one way or another.

There is some evidence that spatial memories may also be viewpoint dependent. Easton and Sholl (1995) found that judgments of relative direction were affected by imagined translations as well as by imagined rotations. For example, suppose an observer had learned the layout in Fig. 2 from the point of view marked $0^{\circ}$. The instruction, "Imagine you are standing where you learned the layout, facing the shoe" does not require imagined translation 

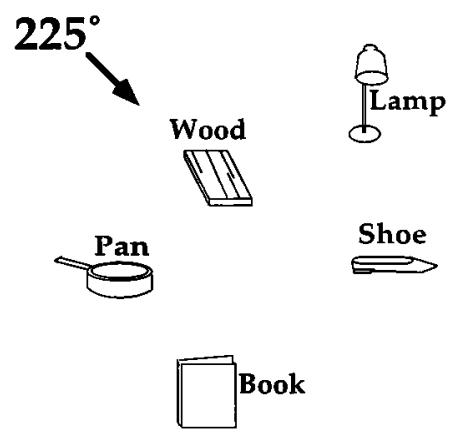

\section{Shoe}
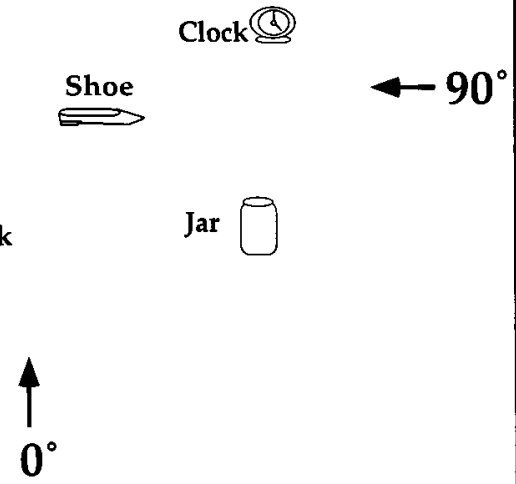

FIG. 2. One of the displays of objects used by Shelton and McNamara (1997a, 1997b) and in Experiment 1. Arrows indicate the three viewing positions used in Experiment 1.

and requires only minimal rotation, whereas the instruction, "Imagine you are standing at the shoe facing the lamp" requires imagined translation but not imagined rotation. In Easton and Sholl's experiments, judgments of the first kind were easier than judgments of the second kind, although the effects were small. Other studies have not revealed effects of imagined translation (e.g., Rieser, 1989; Presson \& Montello, 1994). In our opinion, the verdict on this issue is not yet in.

These one- and two-view studies represent a limited case, however. More than two views of a layout may be required to develop an orientationindependent representation (Biederman \& Gerhardstein, 1993; Marr, 1982; 
Presson, DeLange, \& Hazelrigg, 1987). Presson et al. (1987) had people learn four-point paths (e.g., Fig. 1) kinesthetically. Blindfolded participants moved through the paths while walking normally (allowing multiple orientations) or while maintaining a constant body orientation (incorporating forward walking, side-stepping, and backward walking). The results indicated that participants who experienced multiple orientations at study could perform judgments of relative direction equally well from positions aligned and contra-aligned with the original orientation of the display. Those who maintained a single orientation at study were slower and less accurate at contraaligned judgments than at aligned judgments. In similar work, Hintzman, O'Dell, and Arndt (1981) allowed participants to view a circular display of objects by spinning in a chair. Their results also indicated that participants were able to use the spatial information in an orientation-independent manner. Results such as these have been used to argue that experiencing multiple views allowed participants to form orientation-independent representations.

One problem with these studies, however, is that they have not controlled the number of orientations that participants were allowed to experience. For example, during kinesthetic learning, Presson et al.'s (1987) participants had equivalent experience with nearly all possible orientations. Hence, it is possible that their memories of the layouts consisted of multiple, orientation-dependent representations. Behaviorally, an exhaustive collection of orientation-dependent representations is indistinguishable from an orientation-independent representation (e.g., Tarr \& Pinker, 1989). One of the goals of the present experiments was to determine whether people could construct an orientation independent representation if they were allowed to learn a spatial layout from three views.

\section{Environmental Reference Systems}

In a previously published experiment (Shelton \& McNamara, 1997a), we required observers to learn two orthogonal views of a spatial display $\left(0^{\circ}\right.$ and $90^{\circ}$ in Fig. 2). Afterward, the participants were required to make judgments of relative direction using their memories of the layout. Judgments were faster and more accurate when the imagined heading was parallel to either of the study views (e.g., "at the book facing the wood" or "at the shoe facing the pan') than when it was not parallel to a study view (e.g., " at the wood facing the jar'). We concluded that our results supported a theory of spatial memory in which multiple views produced multiple orientationdependent representations in memory.

One feature of that experiment warrants additional consideration, namely the choice of learned views. Participants learned two orthogonal views that were parallel to the cardinal axes as defined by the walls of the room (see Fig. 2). One important question is whether such alignment with the geometry of the room played a role in how the spatial layout was mentally represented. Although several experiments have been aimed at determining whether spa- 
tial memories are orientation-dependent or orientation-independent, very little is known about the influence of the surrounding environment on how interobject spatial relations are represented in memory.

One domain in which this issue has been investigated systematically is spatial memory development. Acredolo (1978, 1979; Acredolo \& Evans, 1980), in particular, examined how children use egocentric and environmental reference systems. In a series of studies, she trained children to make a particular response relative to both an egocentric and an environmental reference system. For example, children learned to make a head turn to a window on the left when presented with a tone. The children were then moved so that the window was on the opposite side. After moving, the tone was again presented to see if the child would make the egocentric response (turn head to the left again) or the environmental response (turn head to the window, now on the right). Acredolo (1978) found that very young children made egocentric responses, but between 6 and 16 months of age children began using the environmental information, turning to the landmark (window). Additional experiments suggested that the use of such information was facilitated when landmarks were made more salient (Acredolo \& Evans, 1980) or when children were placed in familiar environments (Acredolo, 1979).

Many of the environments in which we behave are highly structured (e.g., rectangular rooms), providing information that might be used to reference locations of objects. Hermer and Spelke $(1994,1996)$ have studied the role of such geometric properties in the spatial representations of young children and adults. After learning the locations of objects in a rectangular room, participants were disoriented. The participants were then asked to orient to the corner in which a particular object had been located originally. In addition to the geometric information from the walls of different lengths, nongeometric information was provided. For example, if one of the short walls was blue and all remaining walls were white, then the geometrically similar corners could be distinguished by the location of the blue wall. The results indicated that young children oriented to the geometrically identical corners of the room equally (that is, the correct location and its opposite), whereas adults used both the geometric and nongeometric properties (blue wall) to reorient. These results suggest that the geometry of the environment plays an important role in spatial representation early in development. The use of additional, nongeometric properties appears to come later in development (but see Learmonth, Newcombe, \& Huttenlocher, 1998).

Previous work in our laboratory has also suggested that the geometry of the enclosing room, and more specifically alignment with the walls of the room, might influence how adults mentally represent space. In an experiment reported by Shelton and McNamara (1997b), observers learned two views of a spatial layout similar to the one depicted in Fig. 2. Half the participants learned two views that were aligned with the walls of the room (e.g., in Fig. $2,0^{\circ}$ and $90^{\circ}$ ), and half the participants learned two views that were misa- 
ligned with the walls (e.g., $45^{\circ}$ and $135^{\circ}$ ). Performance in subsequent judgments of relative direction indicated that participants who learned the aligned views of $0^{\circ}$ and $90^{\circ}$ represented both views in memory, but participants who learned the misaligned views of $45^{\circ}$ and $135^{\circ}$ only represented one of the learned views in memory. These results indicated that the structure of the environment interacted with viewpoint in determining how the space was mentally represented. The principal goal of the experiments reported in this article was to explore such effects more systematically.

\section{Overview of the Experiments}

In Experiment 1, we extended the Shelton and McNamara (1997a) experiment by adding a third study view that was misaligned with the walls of the room. This additional view allowed us to compare directly the aligned and misaligned views to determine whether alignment with the room influenced the presence of multiple representations in memory, as suggested by other experiments (Shelton \& McNamara, 1997b).

In Experiments 2-5, we explored the role of the external environment more systematically, using a paradigm motivated by the work of Pani and his colleagues (Pani \& Dupree, 1994; Pani, Williams, \& Shippey, 1995). Pani and Dupree (1994) manipulated the correspondence among three spatial reference systems: (a) the permanent spatial environment defined by the gravitational upright and the structure provided by the floor, ceiling, and walls; (b) a local spatial environment defined by a wooden box in which the stimuli were mounted; and (c) the perspective of the observer defined by the orientation of the head (assuming forward gaze direction). People were best at imagining and perceiving rotational motion when the axis of rotation corresponded to the environmental upright; however, the local environment also contributed to the ease of imagined rotation. In Experiments 2-5, we manipulated the relations among the observer's point of view, the "global" environmental reference system defined by the walls of the enclosing room, and a "local" environmental reference system defined by the borders of a large mat on which the objects were placed. Finally, in Experiments 6 and 7, we explored the influence of the external environment by removing it. Participants learned the locations of objects in a "spatial ganzfeld," in which the surrounding environment did not provide a salient reference system.

In summary, the major findings from the experiments were these: First, there was no evidence that a small number $(\leq 3)$ of stationary views of a spatial layout led to the formation in memory of an orientation-independent mental representation. Second, the locations of objects seemed to be represented in memory in terms of the viewer's perspective and the external environment, at least when the environment provided a salient system of reference. Indeed, under appropriate conditions, if a study view was misaligned with the walls of the surrounding room, it was no more accessible in memory than novel views, even when it was the first view that participants had of 
the space. Third, and finally, when the environment did not provide a salient, external system of reference, observers represented the space in terms of the system of reference defined by their first encounter with the space.

\section{EXPERIMENT 1}

Participants in Experiment 1 experienced a room-sized spatial layout from three views. Two of these views were aligned with the walls of the room, and one was misaligned. Hence, two questions could be asked: First, Would three views lead to the construction in memory of an orientation-independent mental representation? and second, Would alignment with the walls of the room influence how a view was mentally represented?

\section{Method}

\section{Participants}

Twenty-four undergraduates (12 male and 12 female) participated as part of a requirement or extra credit in their summer psychology courses.

\section{Materials and Design}

The materials were similar to those used by Shelton and McNamara (1997a), with a few minor modifications. We used two configurations of seven objects each. Objects were selected with the restrictions that they be visually distinct, fit within approximately $1 \mathrm{ft}^{2}$, share no primary semantic associations, and have monosyllabic names. Objects were placed on the floor at one end of a $5 \times 7 \mathrm{~m}$ room; the collection of objects occupied a space of approximately $3 \times 3 \mathrm{~m}$. The floor was carpeted, but otherwise bare, except for the objects. Three viewing positions were selected, $0^{\circ}, 90^{\circ}$, and $225^{\circ}$ (shown in Fig. 2). The $0^{\circ}$ and $90^{\circ}$ views are the same aligned views used by Shelton and McNamara (1997a) and the $225^{\circ}$ view is the misaligned view equidistant between $0^{\circ}$ and $90^{\circ}$. Two orders of viewing were used, one beginning at $0^{\circ}$ and moving counterclockwise to $90^{\circ}$ and $225^{\circ}$ and one beginning at $225^{\circ}$ and moving clockwise to $90^{\circ}$ and $0^{\circ}$. This provision was used to keep the amount and location of walking constant across participants while varying whether the first view was aligned or misaligned with the walls of the room.

Each test trial was constructed from the names of three objects in the display and required participants to point to an object as if standing in a particular position within the display; for example, "Imagine you are standing at the jar and facing the shoe; point to the clock.' The first two objects established the imagined vantage point and heading (e.g., jar and shoe) and the third object was the target (e.g., clock). Imagined translations were expected to have small effects relative to the effects of imagined rotation (e.g., Easton \& Sholl, 1995; Presson \& Montello, 1994; Rieser, 1989) and were therefore not examined.

The primary independent variable was imagined heading. Eight equally spaced headings were used. To facilitate exposition, headings were arbitrarily labeled counterclockwise from $0^{\circ}$ to $315^{\circ}$ in $45^{\circ}$ steps beginning with the position labeled $0^{\circ}$ in Fig. 2. For example, $0^{\circ}$ corresponds to all headings oriented in the same direction as the arrow labeled $0^{\circ}$ (e.g., bookwood and jar-clock), $90^{\circ}$ corresponds to all headings oriented with the arrow labeled $90^{\circ}$ (e.g., jar-book and clock-wood), and $270^{\circ}$ corresponds to all headings oriented in the direction opposite to the arrow labeled $90^{\circ}$ (e.g., book-jar and wood-clock).

Pointing direction (the direction of the target object relative to the imagined heading) was varied systematically by dividing the space about a given heading into six areas, front-right 
$\left(0^{\circ}-60^{\circ}\right)$, right $\left(60^{\circ}-120^{\circ}\right)$, back-right $\left(120^{\circ}-180^{\circ}\right)$, and so on. Participants experienced approximately equal instances of each pointing direction with at least one instance for each imagined heading. Participants were given a total of 72 trials, 9 trials at each of 8 imagined headings.

The principal dependent measure was the angular error of the pointing response, measured as the absolute angular difference between the judged pointing direction and the actual direction of the target. Response latencies were also collected to check for speed-accuracy tradeoffs, but because the judgments were not speeded, the most important dependent variable was accuracy.

\section{Procedure}

Four groups were formed by factorially combining configuration and order of views. Participants were randomly assigned to each group with the constraint that each group contain an equal number of males and females.

Learning phase. Before entering the study room, each participant was instructed to learn the objects and their locations for a spatial memory test. The participant was blindfolded and led to the first viewing position (e.g., $0^{\circ}$ or $225^{\circ}$ in Fig. 2 depending on the group). The participant viewed the display for $30 \mathrm{~s}$ before being asked to point to and name each object with eyes closed. Once a participant could accurately generate all names and locations twice, the participant was blindfolded and walked to the second viewing position. Again, the participant viewed the display for $30 \mathrm{~s}$ and had to point to and name the objects two times correctly. This procedure continued for the third viewing position. Before moving from a particular viewing position, participants were offered additional viewing time, and most chose to view the display for 5-10 additional $\mathrm{s}$ at each position.

Test phase. After learning the spatial layout, participants were taken to another room to be tested. The test trials were presented on a Macintosh computer. Participants first received instructions on using the program and four practice trials involving locations on the campus. Each test trial was initiated by the participant and proceeded as follows: A heading and target appeared (e.g., "Imagine you are standing at the jar and facing the shoe. Point to the clock.'") along with a circle and moveable line. The participant used the mouse to position the line on the circle to represent the direction the target would be if the participant were in the imagined position.

\section{Results and Discussion}

For this and all subsequent experiments, only statistically significant effects are reported. Because of the large number of main effects and interactions in the designs, an $\alpha$ level of .01 was used.

Analyses were based on means computed for each participant and each condition (defined below). Mean angular error collapsed across participants is plotted in Fig. 3 as a function of imagined heading and order of viewing. In this and all subsequent experiments, latency showed the same general pattern as angular error, but the effects tended to be smaller and more variable. There was no evidence of speed-accuracy trade-offs.

Two major results are apparent in Fig. 3. First, there was no evidence that participants were able to construct an orientation-independent mental representation from three views. Judgments of relative direction were more accurate for imagined headings oriented with the familiar views of $0^{\circ}$ and $90^{\circ}$ than for other imagined headings. Second, there was little evidence that 


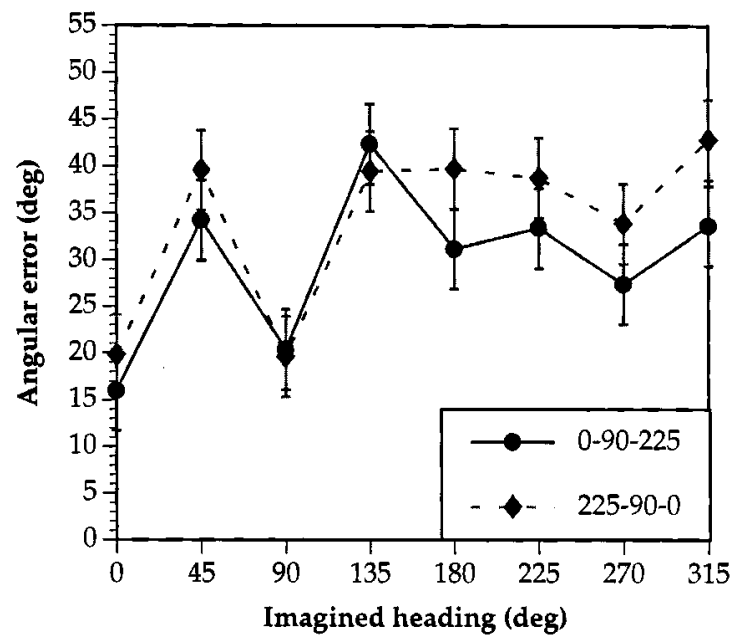

FIG. 3. Angular error in judgments of relative direction as a function of imagined heading and order of viewing in Experiment 1. Participants learned three views of a layout. Two views were aligned with walls of the room $\left(0^{\circ}\right.$ and $90^{\circ}$ in Fig. 2) and one view was misaligned ( $225^{\circ}$ in Fig. 2). Error bars represent \pm 1 standard error of the mean (SEM) calculated from the ANOVA.

the misaligned view from $225^{\circ}$ was represented in memory, even for those participants who learned it first. Judgments of relative direction were no more accurate for imagined headings parallel to the misaligned study view than for imagined headings parallel to novel views.

These conclusions were supported by statistical analyses. Angular error was analyzed using a split-plot factorial ANOVA with terms for configuration (participants learned one of two layouts), order of learning $\left(0^{\circ}-90^{\circ}-\right.$ $225^{\circ}$ vs $\left.225^{\circ}-90^{\circ}-0^{\circ}\right)$, gender, imagined heading $\left(0^{\circ}-315^{\circ}\right.$ in $45^{\circ}$ steps $)$, and pointing direction (front-right, right, back-right, back-left, left, and frontleft). Configuration, order, and gender were between-participants; imagined heading and pointing direction were within-participant.

The effect of imagined heading was significant, $F(7,112)=17.74$. The interaction between imagined heading and order of viewing was not reliable. Performance for imagined headings oriented with the study views of $0^{\circ}$ and $90^{\circ}$ was substantially better than performance for novel imagined headings, $F(1,112)=94.92$. However, performance for imagined headings of $225^{\circ}$ did not differ from performance for novel headings, $F(1,112)=0.02$, and was significantly less accurate than performance for imagined headings of $0^{\circ}$ and $90^{\circ}, F(1,112)=42.55$.

It is noteworthy that performance on novel imagined headings aligned with the walls of the room (e.g., $180^{\circ}$ and $270^{\circ}$ ) was better than performance on novel imagined headings misaligned with the walls of the room (e.g., 
$135^{\circ}$ and $315^{\circ}$ ). This effect was reliable, $F(1,112)=9.29$ (the heading of $45^{\circ}$ was excluded from these comparisons to equate average angular distance from the study views). This effect may occur because the aligned novel views are also opposite to study views (e.g., Diwadkar \& McNamara, 1997; Roskos-Ewoldsen et al., 1998; Vetter, Poggio, \& Bülthoff, 1994). Another possible explanation is that alignment of the study views with the reference system defined by the walls of the room facilitated generalization to novel aligned headings. The present study cannot distinguish between these explanations.

The effect of pointing direction was not significant; however, the interaction between pointing direction and imagined heading was significant, $F(35$, $560)=2.97$. This result appeared to stem from increased difficulty in pointing to objects behind the observer at certain novel headings and did not alter the primary conclusions about the effect of imagined heading.

In summary, the results of Experiment 1 showed that observers were not able to construct an orientation-independent mental representation from three static views. Performance was better for headings oriented with familiar views $\left(0^{\circ}\right.$ and $\left.90^{\circ}\right)$ than for headings oriented with unfamiliar views of the space. The results further indicated that not all study views were equally accessible in memory. Performance on trials in which the imagined heading was oriented with the study view that was misaligned with the walls of the room (viz., $225^{\circ}$ ) was no better than performance on trials in which the imagined heading was oriented with a novel view of the space and was much less accurate than performance on trials in which the imagined heading was oriented with the study views that were aligned with the walls of the room (viz., $0^{\circ}$ and $90^{\circ}$ ). This pattern occurred even when the misaligned view of $225^{\circ}$ was learned first and indicates that the reference system defined by the room provided a powerful cue for organizing memory of the space.

These results suggest that the two aligned views were represented in memory with greater fidelity than was the misaligned view. We know from previous studies (e.g., Roskos-Ewoldsen et al., 1998) that a misaligned view will be represented in memory if it is learned in isolation. Participants in the present experiment did not know that they would be learning more than one view of the layout. Therefore, we can assume that participants who learned the misaligned view first would have shown better performance on this view relative to novel views had it been the only view learned. In the present experiment, the misaligned view appears to have been "forgotten" in the context of the two aligned views. Explanations for this forgetting are more thoroughly addressed under General Discussion. In general, these results indicate that the representation of the space is dynamic during the learning phase of the study; that is, how the space is represented can be altered even after a view has been learned to criterion.

The results of Experiment 1 called for a more systematic investigation of the possible interactions among the reference systems determined by the 
viewer's perspective and the external environment. To this end, in Experiments $2-5$, we manipulated the alignment of the viewer's perspective, the global environmental reference system defined by the room, and a local environmental reference system defined by a large mat on which the objects were placed (e.g., Pani \& Dupree, 1994). Multiple environmental reference systems may play a role in many common activities. For example, when driving a car, it is necessary to monitor the global reference system defined by the environment through which we are traveling, as well as the local reference system defined by the car itself. As such, the inclusion of a local reference system in our study allowed us to examine competition among potential environmental reference systems and their interaction with the viewer's point of view. The major conditions are illustrated in Fig. 4. The global and local reference systems were either congruent or incongruent (Figs. 4a and 4b, respectively), and observers learned one or two views of the layout $\left(0^{\circ}\right.$ or $135^{\circ}$ or both $)$.

\section{EXPERIMENT 2}

Experiment 2 provides the simplest case for examining how environmental reference systems affect the representation of a single view in memory. In this experiment, the local and the global reference systems were congruent (Fig. 4a) and observers learned one view of the layout, $0^{\circ}$ or $135^{\circ}$. Because participants only learned one view of the space, we anticipated that the studied view would be mentally represented even when it was misaligned with global and local environmental reference systems.

\section{Method}

Participants. Twenty-four undergraduates (12 male and 12 female) participated for partial fulfillment of a requirement for their introductory psychology courses.

Materials and procedures. The materials and procedures were similar to those used in Experiment 1 . The same object configurations were used, but there were some important differences in the layout. First, the objects were placed on a $3.3 \times 3.3 \mathrm{~m}$ mat on the floor of the room. The mat was oriented to be congruent with the room (see Fig. 4a). Second, participants learned one layout of objects from a single view, either $0^{\circ}$ or $135^{\circ}$. Four groups of participants were formed from the factorial combination of viewing position (aligned vs misaligned) and configuration. Study and test procedures were conducted as in Experiment 1.

\section{Results and Discussion}

Analyses were based on means computed for each participant and each condition (defined below). Mean angular error collapsed across participants is plotted in Fig. 5 as a function of imagined heading and viewing position $\left(0^{\circ}\right.$ or $\left.135^{\circ}\right)$.

The major result, shown in Fig. 5, was that performance was dependent on orientation, as predicted. People who learned the aligned view at $0^{\circ}$ were more accurate for imagined headings oriented with $0^{\circ}$ than for novel imag- 
SYSTEMS OF SPATIAL REFERENCE
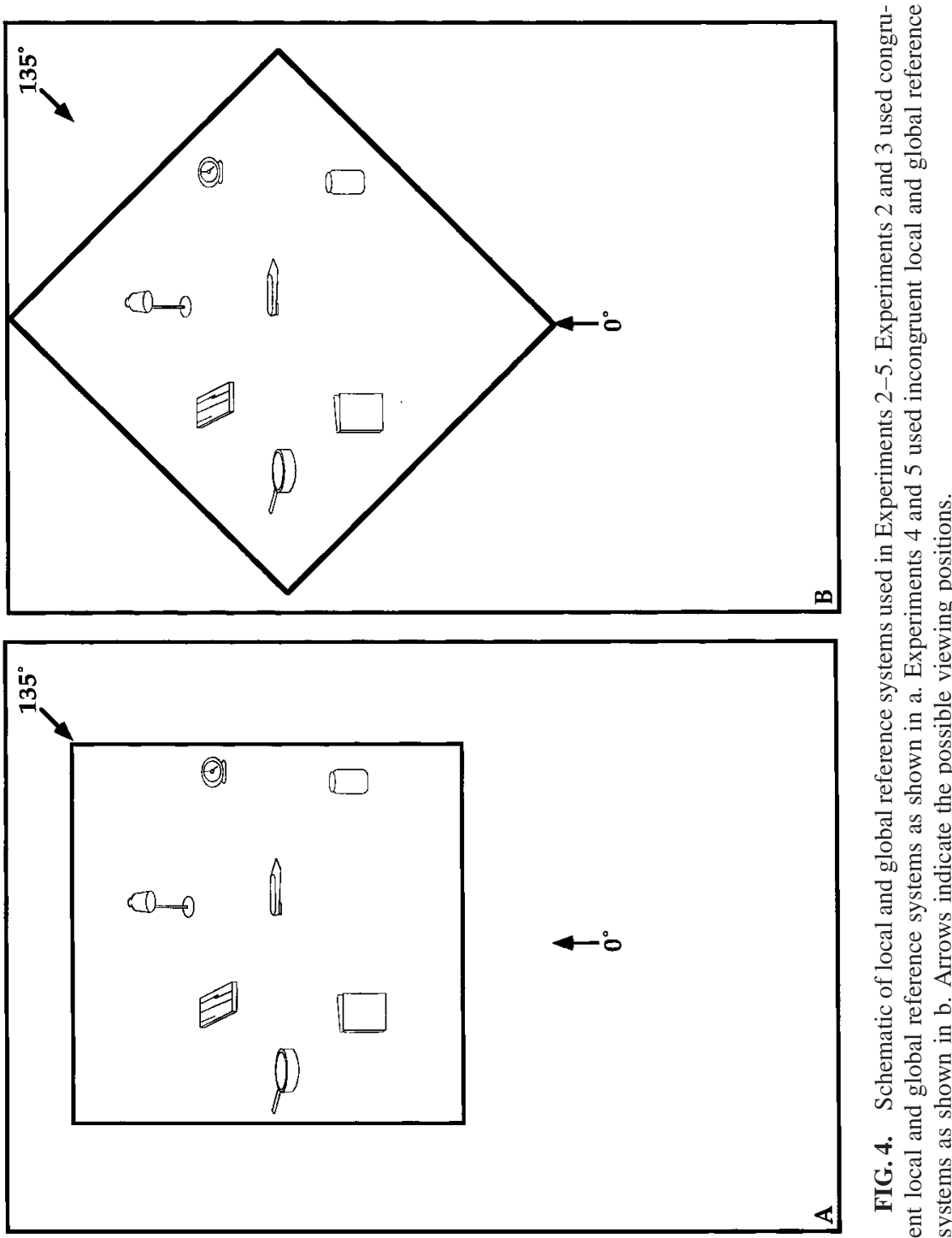

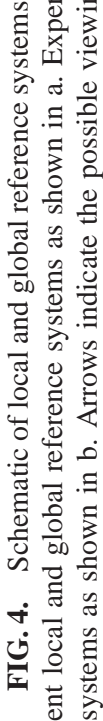




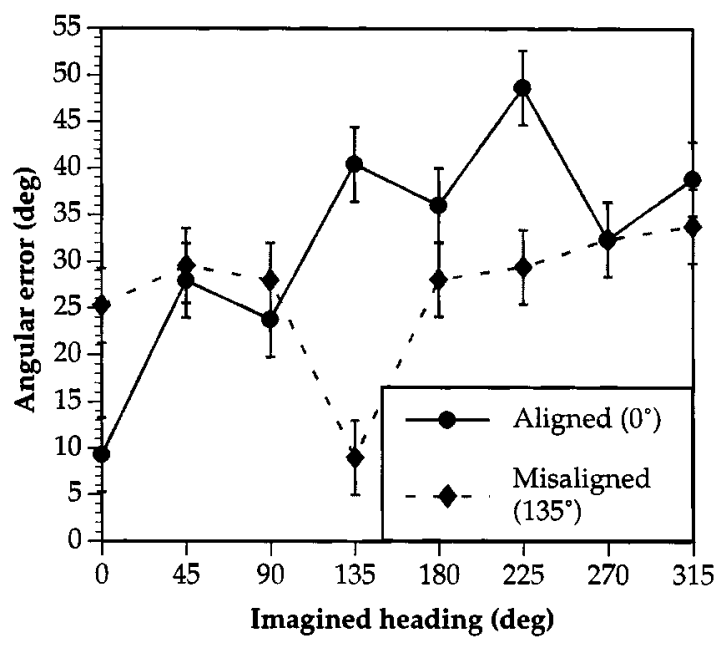

FIG. 5. Angular error in judgments of relative direction ( $\pm S E M)$ as a function of imagined heading and viewing position in Experiment 2. Local and global reference systems were congruent. Participants learned one view of a layout; this view was aligned with both environmental reference systems $\left(0^{\circ}\right)$ or misaligned with both $\left(135^{\circ}\right)$.

ined headings, whereas people who learned the misaligned view at $135^{\circ}$ were more accurate for imagined headings oriented with $135^{\circ}$ than for novel imagined headings. Moreover, learning the aligned view at $0^{\circ}$ produced some benefit for novel imagined headings aligned with the room and the mat relative to novel imagined headings misaligned with the room and the mat. This benefit for novel aligned views was not evident when people learned a misaligned view at study.

Statistical analyses supported these conclusions. Angular error was analyzed using split-plot ANOVA with terms for configuration, viewing position, gender, imagined heading, and pointing direction. Configuration, viewing position, and gender were between-participants; imagined heading and pointing direction were within-participant.

The effect of imagined heading was significant, $F(7,112)=12.13$, as was the interaction between viewing position and imagined heading, $F(7$, $112)=13.47$. Participants who learned the view from $0^{\circ}$ were most accurate on trials in which the imagined heading was oriented with $0^{\circ}, F(1,112)=$ 74.99 , whereas participants who learned the view from $135^{\circ}$ were most accurate on trials in which the imagined heading was oriented with $135^{\circ}, F(1$, 112) $=46.12$.

The comparison of novel aligned headings $\left(90^{\circ}\right.$ and $\left.270^{\circ}\right)$ to novel misaligned headings $\left(45^{\circ}\right.$ and $\left.225^{\circ}\right)$ contrasted by group was significant, $F(1$, 112 ) $=7.50$ (headings of $180^{\circ}$ and $315^{\circ}$ were excluded from this comparison to equate average distance from the study view). This result suggests that people who studied an aligned view ( $0^{\circ}$ group) showed an advantage for 
novel aligned headings over novel misaligned headings, whereas no such advantage emerged for those people who studied a misaligned view (135 group).

The main effect of pointing direction was also significant, $F(5,80)=$ 5.11. Results indicated that pointing to the front was more accurate than pointing to the sides or to the back.

In summary, the results of Experiment 2 showed that with a single view at study, participants had best access to a single view in memory. They further suggested that alignment of the study view with environmental reference systems could influence the accuracy with which certain novel headings were accessed. When the study view was aligned with the two environmental reference systems, participants performed more accurately at aligned novel headings than at misaligned novel headings. When the study view was misaligned with the environmental reference systems, no such benefit was seen for aligned novel headings. In other words, having an aligned study view produced a sawtooth pattern across novel headings, whereas having a misaligned study view produced a smooth pattern across novel headings. This pattern of results suggests that alignment with the environmental reference systems influenced the inferential processes necessary to access novel views.

\section{EXPERIMENT 3}

Experiment 3 extends Experiment 2 to address the role of alignment versus misalignment with multiple views. The global and the local reference systems were congruent and participants learned two views. The views included one aligned view $\left(0^{\circ}\right)$ and one misaligned view $\left(135^{\circ}\right)$. This experiment tests a possible explanation of the finding in Experiment 1 that the misaligned view was represented poorly in memory. Recall that in Experiment 1, participants learned two aligned views and one misaligned view. It is possible that participants were biased by this discrepancy in frequency to notice and exploit alignment with the walls of the room when learning the locations of objects and to ignore the misaligned view. If the discrepancy in frequency alone accounts for the results of Experiment 1, then the aligned and the misaligned view should be mentally represented equally well in Experiment 3. However, if alignment with environmental systems of reference determines, at least in part, whether a view will be mentally represented, then we might expect the aligned view to be represented with greater fidelity than the misaligned view in Experiment 3.

\section{Method}

Participants. Twenty-four undergraduates (12 male and 12 female) participated as partial fulfillment of a requirement for their introductory psychology courses.

Materials and procedures. Materials and procedures were identical to Experiment 2, except that participants learned two views: One view $\left(0^{\circ}\right)$ was aligned with the global and the local system of reference and one view $\left(135^{\circ}\right)$ was misaligned with these systems of reference. The 
order of viewing $\left(0^{\circ}-135^{\circ}\right.$ and $\left.135^{\circ}-0^{\circ}\right)$ was counterbalanced across participants, resulting in four groups based on configuration by order. Participants were randomly assigned to the groups.

\section{Results and Discussion}

Analyses were based on means computed for each participant and each condition (defined below). Mean angular error collapsed across participants is plotted in Fig. 6 as a function of imagined heading and order of viewing $\left(0^{\circ}-135^{\circ}\right.$ or $\left.135^{\circ}-0^{\circ}\right)$.

The major result, shown in Fig. 6, was that performance was most accurate for imagined headings oriented with the aligned study view of $0^{\circ}$ regardless of whether participants learned the $0^{\circ}$ view or the $135^{\circ}$ view first. Moreover, there was little evidence that the misaligned view at $135^{\circ}$ was represented in memory, even for those participants who learned this view first.

Statistical analyses supported these conclusions. Angular error was analyzed using split-plot ANOVA with terms for configuration, order of viewing, gender, imagined heading, and pointing direction. Configuration, order of viewing, and gender were between-participants; imagined heading and pointing direction were within-participant.

The effect of imagined heading was significant, $F(7,112)=15.74$. As shown in Fig. 6, regardless of which study view participants learned first, performance was most accurate for imagined headings oriented with $0^{\circ}$. Indeed, there was no evidence that the view from $135^{\circ}$ was represented at all: Performance on headings oriented with the view of $135^{\circ}$ was not different

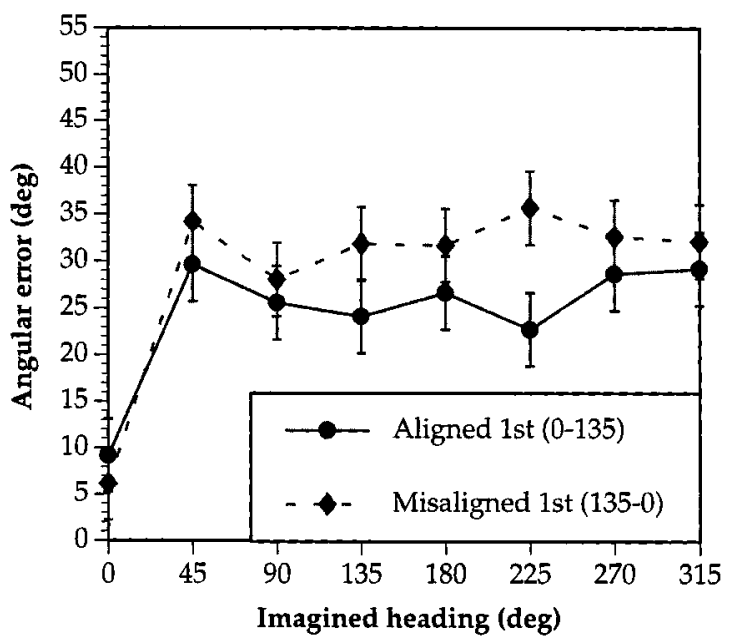

FIG. 6. Angular error in judgments of relative direction $( \pm S E M)$ as a function of imagined heading and order of viewing in Experiment 3. Local and global reference systems were congruent. Participants learned two views. One view $\left(0^{\circ}\right)$ was aligned with both environmental reference systems and one view $\left(135^{\circ}\right)$ was misaligned with both. 
from performance on novel headings, $F(1,112)=0.66$. No obvious advantage for novel aligned headings was found, $F(1,112)=1.13$.

The main effect of pointing direction was also significant, $F(5,80)=$ 6.56. Results were consistent with previous experiments, with front more accurate than sides and sides more accurate than back.

These results indicate that, regardless of which view was learned first, participants seemed to represent only the aligned study view; there was no evidence in judgments of relative direction that participants even saw the view from $135^{\circ}$. Collectively, the results of Experiments 1 and 3 indicate that misaligned study views are only weakly represented in memory (if they are represented at all) when they are experienced in the context of at least one other view that is aligned with environmental reference systems. Given the results of Experiment 2 showing that a misaligned study view was represented in memory when it was learned alone, the results of Experiment 3 again demonstrate that participants were modifying their representations of the space throughout the study phase, based on the interaction between their egocentric perspective and the environmental reference systems available.

In Experiment 2, an advantage emerged for novel headings aligned with environmental reference systems when the study view was also aligned. No such advantage was observed in this experiment, however, suggesting that it only emerges under certain conditions. Perhaps, the salience of the environmental reference systems was diminished by the presence of the misaligned view. We consider alternative explanations of this effect under General Discussion.

\section{EXPERIMENT 4}

Experiments 4 and 5 extended Experiments 2 and 3 by placing the global and the local reference systems in competition (see Fig. 4b) in order to explore their relative contributions. In Experiment 4, observers again learned a single view of the layout. The study view was therefore aligned with either the global reference system or the local reference system.

\section{Method}

Participants. Twenty-four undergraduates (12 male and 12 female) participated in return for extra credit in a summer school course.

Materials and procedures. Materials and procedures were identical to Experiment 2 except for the orientation of the large mat on the floor. The mat was placed at a $45^{\circ}$ angle to the walls of the room (see Fig. 4b). The orientation of the configuration was maintained relative to the room, thus the views of the objects were identical to those in Experiment 2. Again participants learned one spatial layout from a single view, either $0^{\circ}$ or $135^{\circ}$. The $0^{\circ}$ viewing position was aligned with the global reference system and the $135^{\circ}$ position was aligned with the local reference system. Four groups were formed from the factorial combination of viewing position (global-aligned and local-aligned) and configuration. Participants were randomly assigned to these groups. 


\section{Results and Discussion}

Analyses were based on means computed for each participant and each condition (defined below). Mean angular error collapsed across participants is plotted in Fig. 7 as a function of imagined heading and viewing position $\left(0^{\circ}\right.$ or $\left.135^{\circ}\right)$.

The major result, shown in Fig. 7, was that performance was again dependent on orientation as predicted. People who learned the globally aligned view at $0^{\circ}$ were more accurate at imagined headings oriented with $0^{\circ}$ than at novel imagined headings, whereas people who learned the locally aligned view at $135^{\circ}$ were more accurate at imagined headings oriented with $135^{\circ}$ than at novel imagined headings. Moreover, people who learned the locally aligned view $\left(135^{\circ}\right)$ showed some benefit for novel imagined headings that were also aligned with the local reference system relative to novel imagined headings aligned with the global environment. No such benefit for globally aligned novel views emerged for the people who learned the globally aligned view $\left(0^{\circ}\right)$.

Statistical analyses supported these conclusions. Angular error was analyzed using a split-plot ANOVA with terms for configuration, viewing position, gender, imagined heading, and pointing direction. Configuration, viewing position, and gender were between-participants; imagined heading and pointing direction were within-participant.

The effect of imagined heading was significant, $F(7,112)=5.52$, as was the interaction between imagined heading and viewing position, $F(7,112)=$

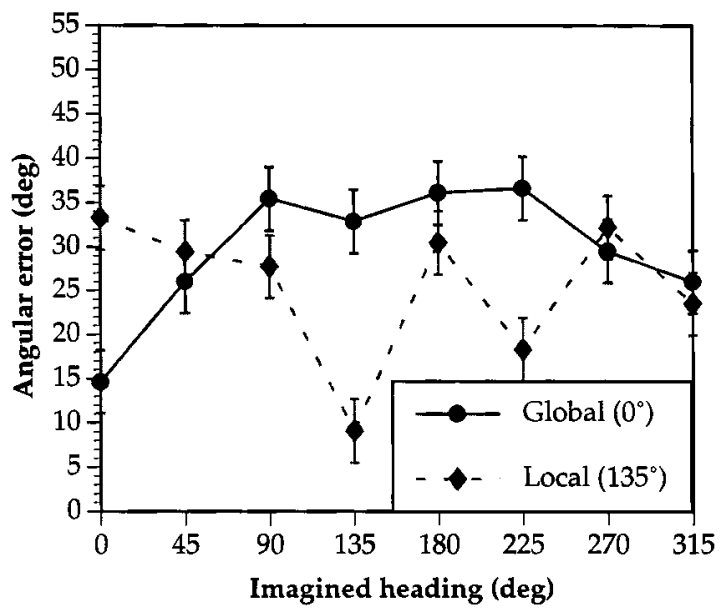

FIG. 7. Angular error in judgments of relative direction $( \pm S E M)$ as a function of imagined heading and viewing position in Experiment 4. Local and global reference systems were incongruent. Participants learned one view of a layout; this view was aligned with the global $\left(0^{\circ}\right)$ or the local $\left(135^{\circ}\right)$ reference system. 
13.71. As shown in Fig. 7, participants who learned the $0^{\circ}$ study view were most accurate on headings oriented with $0^{\circ}, F(1,112)=40.18$, whereas participants who learned the $135^{\circ}$ view were most accurate on headings oriented with $135^{\circ}, F(1,112)=48.00$.

An interaction contrast that tested whether the difference between locally aligned novel headings $\left(45^{\circ}\right.$ and $\left.225^{\circ}\right)$ and globally aligned novel headings $\left(90^{\circ}\right.$ and $\left.270^{\circ}\right)$ was the same for both groups was not significant (this contrast controlled for distance to the study view). This contrast was probably not statistically significant because of the absence of a difference at the $45^{\circ}$ heading. A comparison that ignored distance to the study view showed that performance was better on the locally aligned novel headings $\left(45^{\circ}, 225^{\circ}\right.$, and $\left.315^{\circ}\right)$ than on the globally aligned novel headings $\left(90^{\circ}, 180^{\circ}\right.$, and $\left.270^{\circ}\right)$ for the locally aligned group (viewpoint of $\left.135^{\circ}\right), F(1,112)=9.57$, whereas there was no difference between these sets of novel headings for the globally aligned group (viewpoint of $0^{\circ}$ ).

The main effect of pointing direction was also significant, $F(5,80)=$ 11.79. Results were consistent with previous experiments, with pointing to the front more accurate than pointing to the sides, and pointing to the sides more accurate than pointing to the back.

These results suggest that when the local and the global reference systems are incongruent, alignment of the study view with the local system may not be equivalent to alignment with the global system. Although performance on headings oriented with the study view (either locally aligned or globally aligned) was always better than performance on novel headings, alignment with the local reference system at study also produced some facilitation at test on novel headings aligned with the local reference system. Alignment at study with the global reference system did not produce a corresponding benefit. This unexpected benefit for the local reference system suggests that it may take precedence over the global reference system.

\section{EXPERIMENT 5}

Experiment 5 was designed to examine the relative importance of the local and the global reference systems when multiple views were learned. The global and the local reference systems were incongruent as in Experiment 4 , but participants now learned two views of the layout. Each participant learned a globally aligned view $\left(0^{\circ}\right)$ and a locally aligned view $\left(135^{\circ}\right)$. The key question was whether alignment with any environmental reference system facilitated representation of a view or if one of the reference systems was more salient than the other. From the single view studies, it might appear that the local reference system takes precedence over the global reference system given that alignment locally at study yielded higher accuracy at locally aligned novel headings than other novel headings. If this benefit is indicative of dominance of the local reference system, then we might expect 
only the locally aligned view to be represented with high fidelity. Alternatively, if participants can exploit information from either reference system, we might expect the locally and the globally aligned study views to be represented equally well in memory.

\section{Method}

Participants. Twenty-four undergraduates (12 male and 12 female) participated as partial fulfillment of a requirement for their introductory psychology courses or for extra credit in a summer school course.

Materials and procedures. Materials and procedures were identical to the previous experiments, except that the global and the local reference systems were incongruent and participants learned two views of the layout. One view $\left(0^{\circ}\right)$ was aligned with the global but misaligned with the local system of reference; the other view $\left(135^{\circ}\right)$ was misaligned with the global but aligned with the local system of reference. The order of viewing $\left(0^{\circ}-135^{\circ}\right.$ and $\left.135^{\circ}-0^{\circ}\right)$ was counterbalanced, resulting in four groups based on configuration by order. Participants were randomly assigned to these groups.

\section{Results and Discussion}

Analyses were based on means computed for each participant and each condition (defined below). Mean angular error collapsed across participants is plotted in Fig. 8 as a function of imagined heading and order of viewing $\left(0^{\circ}-135^{\circ}\right.$ or $\left.135^{\circ}-0^{\circ}\right)$.

The major results were these: First, performance was more accurate for imagined headings oriented with the two study views than for novel imagined headings. Regardless of the order of viewing, judgments of relative direction

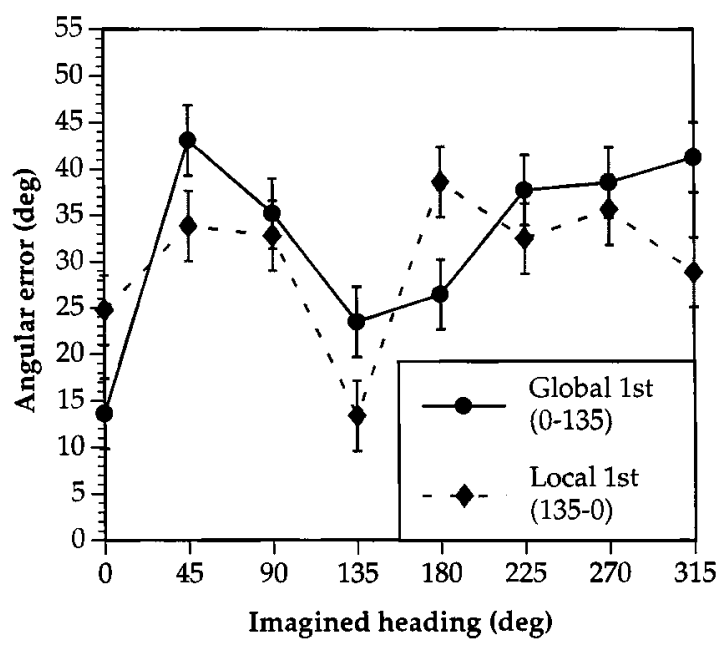

FIG. 8. Angular error in judgments of relative direction $( \pm S E M)$ as a function of imagined heading and order of viewing in Experiment 5. Local and global reference systems were incongruent. Participants learned two views. One view $\left(0^{\circ}\right)$ was aligned with the global reference system and one view $\left(135^{\circ}\right)$ was aligned with the local reference system. 
were more accurate for imagined headings corresponding to $0^{\circ}$ and $135^{\circ}$ than for novel imagined headings. Second, there appeared to be a primacy effect, with better performance on the first study view than on the second study view. People who learned $0^{\circ}$ first were more accurate at $0^{\circ}$ headings than at $135^{\circ}$ headings, whereas people who learned $135^{\circ}$ first were more accurate at $135^{\circ}$ headings than at $0^{\circ}$ headings.

Statistical analyses supported these conclusions. Angular error was analyzed using a split-plot ANOVA with terms for configuration, order of viewing, gender, imagined heading, and pointing direction. Configuration, order of viewing, and gender were between-participants; imagined heading and pointing direction were within-participant.

The effect of imagined heading was significant, $F(7,112)=17.38$, as was the interaction between imagined heading and order of viewing, $F(7$, $112)=6.10$. As shown in Fig. 8, participants were more accurate on imagined headings oriented with $0^{\circ}$ and $135^{\circ}$ than on novel imagined headings, $F(1,112)=115.15$. The interaction contrast for the two study views by the two viewing orders was significant, $F(1,112)=15.82$, indicating that in each group, performance on the study view experienced first was better than performance on the study view experienced second.

The difference between locally aligned novel headings $\left(45^{\circ}, 225^{\circ}\right.$, and $\left.315^{\circ}\right)$ and globally aligned novel headings $\left(90^{\circ}, 180^{\circ}\right.$, and $\left.270^{\circ}\right)$ varied as a function of which study view was learned first, $F(1,112)=13.21$. The pattern of this crossover interaction indicated that novel headings aligned with the same reference system as the first study view were easier to imagine than were novel headings aligned with the same reference system as the second study view. However, this effect was small and the functions did not show the dramatic saw-tooth pattern observed in Experiments 2 and 4 (Figs. 5 and 7). As in Experiment 3, this diminished effect raises questions about the conditions and processes that might lead to this benefit.

The effect of pointing direction was significant in angular error, $F(5,80)=$ 6.01. Results were consistent with previous experiments, with front more accurate than sides and sides more accurate than back.

These results suggest that multiple representations of the space may be available simultaneously. Performance was better on headings oriented with the study views than on novel headings, and neither the locally aligned view nor the globally aligned view was "preferred"' over the other. There was a small "primacy" effect, such that performance was better on the first study view than on the second. This effect was not observed in Experiment 1 (in the comparison of $0^{\circ}$ to $90^{\circ}$ ), nor was it observed by Shelton and McNamara (1997a). The result indicates that, at least in the conditions of this experiment, there might have been a cost associated with representing the second study view when it was aligned with a different reference system from the first study view. This cost, however, was small, especially relative to the overall difference between familiar and novel headings. 
The major results of Experiments 1-5 were these: Spatial memories were orientation-dependent even when participants were required to learn the layouts from three points of view. Study views that were misaligned with salient environmental reference systems seemed not to be strongly represented in memory if they were experienced in the context of aligned study views. Finally, there was some evidence that novel views were easier to imagine when they were aligned with local reference systems than when they were misaligned with those reference systems.

In Experiments 6 and 7, the effects of egocentric and environmental reference systems were examined by having participants learn the locations of objects in a room that did not have salient external reference systems. A cylindrical room was created in the laboratory in such a way that the walls of the room and the ceiling could not be seen (see Fig. 9). The floor on which objects were located was bare, but for the carpet.

\section{EXPERIMENT 6}

Experiment 6 was designed to investigate how a spatial layout would be mentally represented if it was learned from a single viewpoint, in the absence of salient environmental reference systems. Participants learned one view of the layout, $0^{\circ}$ or $225^{\circ}$.

\section{Method}

Participants. Twenty-four undergraduates (12 male and 12 female) participated as partial fulfillment of a requirement for their introductory psychology courses.

Materials. A 20-sided cardboard room was constructed to form a $3.6 \mathrm{~m}$ diameter cylinder around the display. One panel was equipped to function as a door. The walls of the cylindrical room were $2.25 \mathrm{~m}$ tall, leaving a small (approximately $25-\mathrm{cm}$ ) gap between the top of the wall and the ceiling of the enclosing room. A curtain was hung around the outside of the room, extending approximately $40 \mathrm{~cm}$, to fill the gap. Participants learned the layout from a single view, which corresponded to $0^{\circ}$ or to $225^{\circ}$. Remaining materials were identical to Experiment 1.

Procedures. As in all previous experiments, participants were blindfolded before entering the enclosing room. Inside the room, they were guided into the cylindrical room through the panel door. Once the participant was positioned at the study view, the panel door was closed and the blindfold was removed. The study procedures proceeded as in previous studies until the participant had learned the study view to criterion. After learning the display, participants were blindfolded and led back out of the cylindrical room and the enclosing room. Test procedures were identical to those used in previous experiments.

\section{Results and Discussion}

Analyses were based on means computed for each participant and each condition (defined below). Mean angular error collapsed across participants is plotted in Fig. 10 as a function of imagined heading and viewing position $\left(0^{\circ}\right.$ or $\left.225^{\circ}\right)$.

As predicted, performance was more accurate at imagined headings corre- 


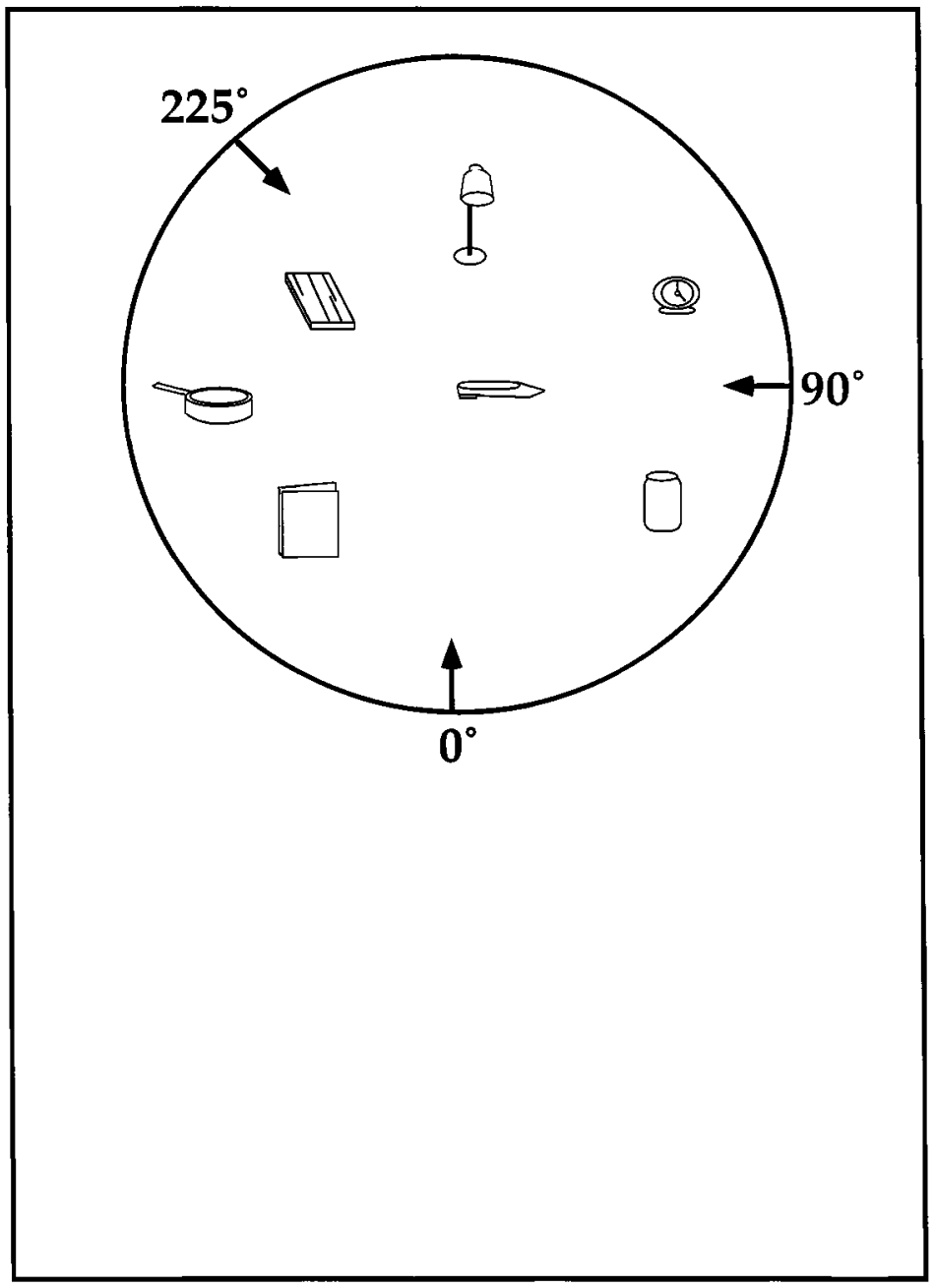

FIG. 9. Schematic of the round room displays used in Experiments 6 and 7. Arrows indicate the possible viewing positions.

sponding to the study view than at novel imagined headings. People who learned the $0^{\circ}$ view were more accurate for imagined headings corresponding to $0^{\circ}$ than for novel imagined headings, whereas people who learned the $225^{\circ}$ view were more accurate for imagined headings corresponding to $225^{\circ}$ than for novel imagined headings.

Statistical analyses supported these conclusions. Angular error was analyzed using a split-plot ANOVA with terms for configuration, viewing position, gender, imagined heading, and pointing direction. Configuration, view- 


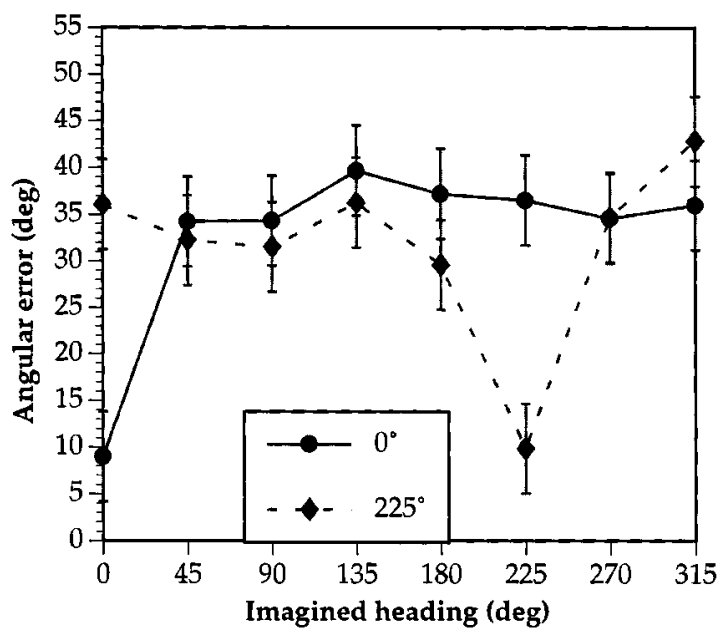

FIG. 10. Angular error in judgments of relative direction $( \pm S E M)$ as a function of imagined heading and viewing position in Experiment 6. Participants learned one view of a layout in a round room $\left(0^{\circ}\right.$ or $225^{\circ}$ in Fig. 9).

ing position, and gender were between-participants; imagined heading and pointing direction were within-participant.

The effect of imagined heading was significant, $F(7,112)=6.65$, as was the interaction between imagined heading and viewing position, $F(7,112)=$ 9.67. As shown in Fig. 10, participants who experienced the $0^{\circ}$ study view were most accurate at trials oriented with $0^{\circ}, F(1,112)=55.51$, whereas participants who experienced $225^{\circ}$ were most accurate at trials oriented with $225^{\circ}, F(1,112)=46.88$.

The effect of pointing direction was also significant, $F(5,80)=7.45$. As in previous experiments, results indicated that front was more accurate than sides and sides were more accurate than back.

Experiment 6 demonstrated orientation-dependent performance in a situation in which the reference system provided by the walls of the room was obscured. Performance was better on headings oriented with the study view than on all novel headings. Among the novel headings, there was limited evidence of facilitation for novel headings directly opposite the study view (e.g., Diwadkar \& McNamara, 1997; Roskos-Ewoldsen et al., 1998; Vetter et al., 1994); however, no other pattern emerged among novel headings. Given that alignment with the environment was not defined in this study, we expected no advantages among novel headings to emerge. These results therefore support the claim that the saw-tooth patterns observed in the previous experiments can be attributed to alignment with environmental reference systems. 


\section{EXPERIMENT 7}

In Experiment 7, participants learned the same layout as in Experiment 6 , but they learned it from three views. Relative to the configurations of objects, which were the same as in the previous experiments, these views corresponded to $0^{\circ}, 90^{\circ}$, and $225^{\circ}$. Hence, observers in Experiment 7 learned the same views of the same layouts as the observers in Experiment 1 . The difference was that the layout existed in a spatial ganzfeld in Experiment 7, whereas the layout was located in a rectangular room in Experiment 1.

\section{Method}

Participants. Twenty-four undergraduates (12 male and 12 female) participated for partial fulfillment of a requirement for their introductory psychology courses.

Materials and procedures. Materials and procedures were identical to those used in Experiment 6 , except for the number of study views. Participants experienced the layout from three views. The order of learning was counterbalanced across participants $\left(0^{\circ}-90^{\circ}-225^{\circ}\right.$ vs $225^{\circ}-$ $\left.90^{\circ}-0^{\circ}\right)$.

\section{Results and Discussion}

Analyses were based on means computed for each participant and each condition (defined below). Mean angular error collapsed across participants is plotted in Fig. 11 as a function of imagined heading and order of viewing $\left(0^{\circ}\right.$ first vs $225^{\circ}$ first $)$.

Two important results are evident in Fig. 11. First, only the first study view seemed to be represented in memory. For participants who learned $0^{\circ}$

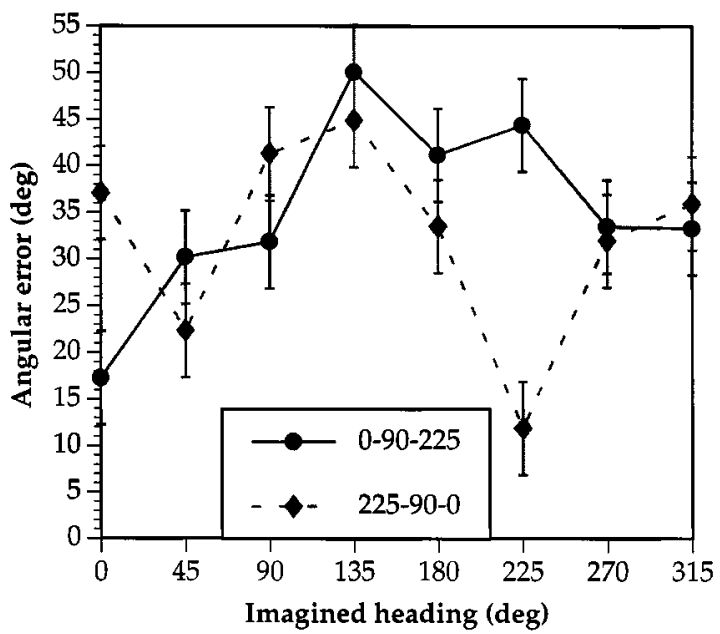

FIG. 11. Angular error in judgments of relative direction ( $\pm S E M)$ as a function of imagined heading and order of viewing in Experiment 7. Participants learned three views of a layout in a round room $\left(0^{\circ}, 90^{\circ}\right.$, and $225^{\circ}$ in Fig. 9). 
first, performance was best on headings oriented with $0^{\circ}$, and there was no savings at $90^{\circ}$ or $225^{\circ}$, and for participants who learned $225^{\circ}$ first, performance was best on headings oriented with $225^{\circ}$, and there was no savings at $90^{\circ}$ or $0^{\circ}$. The second major result was that performance was still orientationdependent, even though participants experienced the layout from three views.

These conclusions were supported by statistical analyses. Angular error was analyzed using a split-plot ANOVA with terms for configuration, order of viewing, gender, imagined heading, and pointing direction. Configuration, order of viewing, and gender were between-participants; imagined heading and pointing direction were within-participant.

The effect of imagined heading was significant, $F(7,112)=6.78$, as was the interaction between imagined heading and order of viewing, $F(7,112)=$ 8.09. Simple main effect contrasts revealed that participants who experienced the $0^{\circ}$ study view first were most accurate on trials oriented with $0^{\circ}, F(1$, $112)=25.69$, and participants who experienced $225^{\circ}$ first were most accurate on trials oriented with $225^{\circ}, F(1,112)=33.29$. Much of this interaction can be accounted for by the interaction contrast of $0^{\circ}$ versus $225^{\circ}$ for the two groups, $F(1,112)=47.56$, suggesting that the difference was due to the study view and not to differences among novel views. There was also evidence of savings in angular error for the heading opposite the first study view, but only for the group that learned $225^{\circ}$ first, $F(1,112)=10.18$.

The effect of pointing direction was also significant, $F(5,80)=8.25$. As in previous experiments, results indicated that front was more accurate than sides and sides were more accurate than back. The interaction between pointing direction and imagined heading was also significant, $F(5,560)=2.60$. This interaction was very small and can be attributed to participants' increased error in pointing behind themselves at certain novel headings; it did not alter the primary conclusions about imagined heading and order of views.

To determine whether the additional study views in Experiment 7 provided any benefit, we compared the error data from each study. The mean angular errors were $32.15^{\circ}$ for Experiment 6 and $33.79^{\circ}$ for Experiment 7, suggesting that additional views did not improve overall performance. More important, the mean discrepancy in angular error between the best view $\left(0^{\circ}\right.$ or $\left.225^{\circ}\right)$ and all remaining views was $25.97^{\circ}$ in Experiment 6 and $21.95^{\circ}$ in Experiment 7. This difference of $4.02^{\circ}$ was not significant, $t(46)=1.00$, indicating that generalization to novel views did not benefit from additional experience.

\section{GENERAL DISCUSSION}

The principal goal of the experiments reported in this article was to determine the relative importance of egocentric and environmental reference systems in representing the spatial structure of the surrounding environment. Experiment 1 was a natural extension of previous experiments conducted in our laboratory (e.g., Shelton \& McNamara, 1997a). Subjects learned the 
locations of objects in a room from three points of view, two of which were aligned with the walls of the room and one of which was in the corner, and therefore misaligned with the walls of the room. Experiments 2-5 examined in more detail the interactions among egocentric and environmental reference systems. Objects were placed on a local reference system defined by a large mat; the orientation of the mat was congruent or incongruent with the global reference system defined by the walls of the room. Participants learned one or two views of the layout; study views were therefore aligned with one, both, or neither reference system. Finally, in Experiments 6 and 7, participants learned one or three views (respectively) of a layout of objects in a cylindrical room. After learning a layout, participants in all experiments made judgments of relative direction using their memories. The results showed that participants' memories were sensitive to egocentric and to environmental reference systems, and these reference systems seemed to interact in complex ways. The specific findings are presented below.

First, in all of the experiments, participants were best able to imagine the spatial structure of the environment from an experienced perspective. There was no evidence that observers were able to construct view-invariant representations, even with up to three study views. Indeed, there was no improvement in performance on novel headings as the number of learned views increased from one to three. The difference in performance between (a) headings oriented with the best study view or views and (b) all remaining headings was $22^{\circ}, 19^{\circ}$, and $20^{\circ}$ for one, two, and three view experiments, respectively. Thus, we did not find any evidence for better overall generalization with more study views. ${ }^{1}$

Second, properties of the external environment had a powerful influence on how participants mentally represented the layout. In Experiments 1 and 3 , the study view that was misaligned with the walls of the room (and in Experiment 3, with the local reference system defined by the mat) appeared to be represented weakly in memory (if at all), even for participants who learned that view first; and in Experiment 7, in which participants learned three views in the round room, only the first of the three study views seems to have been mentally represented (see Figs. 3, 6, and 11).

Third, as shown in Experiment 2, participants were able to learn a spatial layout from a viewpoint that was misaligned with environmental reference systems if it was the only view experienced. It is noteworthy that overall accuracy in judgments of relative direction was at least as high for observers who learned the layout from the misaligned view as for observers who learned the layout from the aligned view (see Fig. 5). This result indicates

\footnotetext{
${ }^{1}$ The category of "best' headings only included those oriented with study views; the category of "all remaining headings" included all novel headings as well as headings oriented with study views when performance on those headings did not differ reliably from performance on novel headings.
} 
that misalignment with environmental reference systems did not interfere with observers' abilities to learn the layout.

Fourth, and finally, novel views aligned with the local reference system were, under certain conditions, easier to imagine than were novel views misaligned with the local reference system. This result was most salient in Experiment 4: Observers who learned the layout from $135^{\circ}$, which was aligned with the edges of the mat but misaligned with the walls of the room, were better able to imagine the layout from headings parallel to $225^{\circ}$ and $315^{\circ}$ than from headings parallel to $0^{\circ}, 90^{\circ}, 180^{\circ}$, and $270^{\circ}$ (see Fig. 7 ; we do not know why headings of $45^{\circ}$ did not show the same savings as $225^{\circ}$ and $315^{\circ}$ ). A similar pattern of results appeared in Experiment 2 for observers who learned the layout from $0^{\circ}$ (see Fig. 5).

Our explanation of these challenging findings relies heavily on principles of form perception proposed by Rock (1973). Rock wanted to know why figures in the frontal plane usually look different when their orientation is changed. A classic example is the square-diamond, which looks like a square when oriented with an edge at the top, but like a diamond with a corner at the top. This problem, of course, is one that vexed Gestalt psychologists early in the 20th century (Köhler, 1940; Goldmeier, 1937). Rotation of a figure in the frontal plane changes two things: orientation with respect to the observer and orientation with respect to the environment. Which of these is most important in determining perceived shape? Rock's investigations showed that for novel figures, changing egocentric orientation had little effect on perceived shape. However, when the orientation of a figure with respect to the environment was changed, the figure was seen as different and often not recognized at all.

For example, in one series of experiments, Rock (1956) designed ambiguous figures so that they had different interpretations in different orientations; for example, in one orientation, one of the figures looked like the profile of an old man, but when rotated $90^{\circ}$, it looked like an outline of the United States. The figures were presented to observers whose heads were tilted $90^{\circ}$. When shown these ambiguous figures with heads tilted, observers typically reported seeing the environmentally upright figure rather than the retinally upright figure. Another way to state this is that the observers saw the shape defined by the environmental frame of reference rather than the shape defined by the egocentric frame of reference; indeed, they ignored the egocentric information to interpret the figure in terms of the environmental information.

In another series of experiments (Rock \& Heimer, 1957), novel figures were first shown to an upright observer in a training session and were later presented in a test period, along with new figures. In the test, the observer's head was tilted, and each old figure was presented twice, once in the environmentally upright orientation and once tilted by the same amount as the observer's head. Note that because the observer's head was tilted at test, the tilted test figures actually matched the egocentric orientation of the study 
figures. The finding was that the environmentally upright figures were recognized better than the environmentally tilted figures; indeed, the environmentally upright figures were recognized as well in this experiment as they were in a control experiment in which the observer maintained an upright head orientation at both study and test.

Rock (1973) concluded that the interpretation of a figure depends on which part or region is assigned "top" and that a change in the assignment of this direction profoundly affects perceived shape. The top of a figure is normally assigned on the basis of the information provided by gravity or the visual frame of reference. Other sources of information can also be used, including egocentric orientation, instructions, intrinsic properties of the figure, and familiarity, but these sources were, according to Rock, typically less salient than environmental sources. Rock's experiments also showed that when there was no salient environmental frame of reference, perceived shape was determined by the figure's orientation with respect to the observer. For example, if a figure was viewed on a horizontal surface through a circular aperture, the egocentrically uppermost region was perceived as the top (Rock \& Heimer, 1957). More recent investigations (e.g., Friedman \& Hall, 1996; McMullen \& Jolicoeur, 1990) have shown that Rock (1973) might have underestimated the importance of retinal orientation in the perception of form. Even so, the general principle - that the perception of form involves the assignment of directions based on a spatial reference system-is sound.

Our conjecture is that just as "top" is assigned to figures in form perception, so too, conceptual "north" is assigned to spatial layouts. We are not claiming of course that "north" corresponds to true or magnetic north; rather, "north" refers to a privileged direction in the environment. This assignment of directions determines how the space is interpreted, and hence, how it is mentally represented.

There is a fundamental difference, though, between form perception and spatial memory: Whereas figures in the frontal plane are oriented in a space with a powerful and salient reference axis, namely gravity, the locations of objects are typically defined in the ground plane, which does not have privileged axes or directions (e.g., humans cannot perceive magnetic fields). We therefore propose that in spatial memory, egocentric reference systems are primary and environmental reference systems are secondary. Although environmental reference systems are secondary, they still play an important role in the interpretation of space. The results of our experiments show that egocentric reference systems that are aligned with salient axes in the environment are preferred to those that are not.

This preference for the aligned views may reflect the naturalness of organization afforded by those views. For example, consider the assignment of a conceptual "north" from an aligned and a misaligned view. In a typical map, the cardinal directions are typically aligned with the edges of the page on which the map is printed. If the room structure were treated the same 
way, then assigning straight-ahead as north in the aligned view would be more consistent with typical organizational principles than assigning the straight-ahead as north in the misaligned view. Thus, when given the option, participants use the more logical assignment. Tversky (1981) has shown that people tend to want their environments to adhere to rectangular properties. Although a misaligned view alone did not produce a deficit in performance, it is possible that when given an aligned view and a misaligned view, the "naturalness" of assigning north in the aligned view made it more preferable for organizing the memory.

To see how these principles explain our results, let us work through the major experimental findings in turn. Consider Experiment 7, in which observers learned three views of a spatial layout in the round room (results in Fig. 11). According to our theoretical framework, when observers were taken to the first viewpoint, they interpreted the spatial structure of the layout in terms of the most salient reference system available, namely their own egocentric perspective. This reference system remained the dominant one, even when participants were moved to the next two points of view, because no other point of view was aligned with a salient axis in the environment. The conjecture is that when participants viewed the layout from the second and the third points of view, they still interpreted the spatial structure of the layout in terms of the reference system defined by the first point of view; conceptual "north", was unchanged. Just as Rock's (1956) observers continued to interpret the old man/U.S. figure as an old man, even with their heads tilted, so too our observers continued to interpret the layout in terms of the initial reference system, even when they were taken to new viewpoints in the environment. The difference is that in the perceptual studies, the dominant frame of reference was established by the environment, but in our spatial memory experiments, the dominant frame of reference was established by the observer's first encounter with the space.

Consider now Experiment 2 in which the local and the global reference systems were congruent and observers learned one view of the space, either from $0^{\circ}$ or from $135^{\circ}$ (results in Fig. 5). Our claim is that observers imposed a spatial reference system on the environment from their first and only point of view and interpreted the spatial structure of the environment in terms of that reference system. Because of the dominance of egocentric over environmental reference systems, this reference system was not supplanted by one based on the local or the global environments.

Now let us consider a more complex case, such as Experiment 3. Observers in this experiment learned the layout from two points of view, one was aligned and the other was misaligned with environmental reference systems (results in Fig. 6). Although half of the participants learned the misaligned view first, their data were indistinguishable from those participants who learned the aligned view first: Only the aligned view was strongly represented. How do we explain this finding? 
Observers who first learned the aligned viewpoint $\left(0^{\circ}\right)$ imposed a reference system on the environment for organizing their memory of the layout. When they moved to the misaligned viewpoint $\left(135^{\circ}\right)$, they still interpreted the layout in terms of the reference system established by the first, aligned view. Hence, performance on judgments of relative direction was best for headings parallel to the aligned view and was no better on headings parallel to the misaligned view than on novel headings.

Observers who first learned the misaligned view $\left(135^{\circ}\right)$ also must have interpreted the space in terms of a reference system defined by that view: We know this must be true because of the results of Experiment 2, in which participants were quite able to learn the layout from $135^{\circ}$. So what happened to this mental representation of the layout? Our conjecture is that when participants were taken to the second viewpoint, they reinterpreted the spatial structure of the layout in terms of a reference system defined by the aligned view because it was aligned with salient axes in the environment. We can only speculate on what happened to the representation created at the first viewpoint: It might have been ignored and forgotten; it also might have been normalized to coincide with the representation created at the aligned point of view. Future research is needed to distinguish these possibilities. The major point is that after moving from a misaligned study view to an aligned study view, observers changed the definition of " north." A new spatial reference system-one that was aligned with the environment - was selected and the spatial layout was reinterpreted in terms of it. The results of Experiment 1 (in Fig. 3), in which observers learned two aligned views and one misaligned view, can be explained in a similar manner.

This phenomenon may be similar to what can happen when people look at an ambiguous figure. At first, an observer may not be aware of what he or she is looking at because the reference system used to interpret the figure is not one that corresponds to a familiar figure. If another person, however, points to a crucial feature or axis ("here's the nose, the eye, ...'"), a meaningful interpretation of the figure may become apparent. We do not think that reinterpretation of a spatial layout according to an aligned viewpoint is as compelling as the perceptual example, but the concept is similar.

In Experiments 2 and 4, half of the participants - the aligned $\left(0^{\circ}\right)$ group in Experiment 2 and the local $\left(135^{\circ}\right)$ group in Experiment 4 -learned the layout from a viewpoint that was aligned with the edge of the mat. One of the interesting outcomes of these studies was that observers were better able to imagine the layout from novel views aligned with the local reference system than from novel views misaligned with the local reference system. In Experiment 2, savings appeared at headings of $90^{\circ}, 180^{\circ}$, and $270^{\circ}$, and in Experiment 4, savings appeared at $225^{\circ}$ and $315^{\circ}$. An inspection of individual subjects' data revealed that this pattern of facilitation for aligned novel headings was not created because different subjects used different edges of the mat to define the primary reference system. In the context of the theory 
we are proposing, these results indicate that participants were able to represent, to some extent, the spatial structure of the layout along axes orthogonal to their egocentric orientation. Put another way, this pattern of results indicates that participants established "east" or "west" (or both) in addition to " "north." The savings at novel aligned headings might have been smaller in Experiment 2 than in Experiment 4 because the local reference system was much less salient in 2 than in 4 (notice, e.g., that the square appears smaller than the diamond in Fig. 4, even though they are the same size). ${ }^{2}$ We do not know why the savings at novel headings did not also appear when observers learned two views, as in Experiment 5.

In a previous article (Shelton \& McNamara, 1997a), we argued that two views of a spatial layout produced two orientation-dependent representations in memory. In light of our new findings and the theoretical framework we have proposed, this explanation may need to be revised. In particular, it is not clear whether additional views produce additional representations or additional information in the same representation. Put another way: When participants seem to have represented the layout from two points of view (e.g., Experiments 1 and 5 of this article), have they formed two representations, each with its own conceptual "north," or have they formed a single representation with conceptual "north" and conceptual "west"? Behavioral data may be insufficient for answering such a question because, in the absence of additional processing assumptions, both models predict the same patterns of performance. However, the viability of our proposed theoretical framework does not require distinguishing multiple representations from a single augmented representation. Its merit rests instead on its ability to explain why some study views seem to be represented in memory and others do not.

One of the important discoveries of these experiments was that participants were not able to construct view-invariant representations of the layouts by learning multiple views; in fact, there was no apparent improvement on novel headings as the number of learned views increased from one to three. It is possible that view-invariant performance or better generalization to novel views would occur if participants were allowed four or more learning views (but for evidence to the contrary, see Diwadkar \& McNamara, 1997).

${ }^{2}$ Savings at novel headings aligned with external frames of reference does not seem to occur in the absence of a local system of reference. It is true that Shelton and McNamara (1997a) obtained evidence of generalization to novel views aligned with a global system of reference after participants learned two aligned views of a spatial layout $\left(0^{\circ}\right.$ and $\left.90^{\circ}\right)$. Unfortunately, these findings cannot be interpreted unambiguously because the aligned novel views were also opposite to the study views (e.g., $180^{\circ}$ vs $0^{\circ}$ and $270^{\circ}$ vs $90^{\circ}$ ). There is evidence in the literature that people can sometimes capitalize on the geometric regularities shared by perspectives of objects and scenes separated by $180^{\circ}$ (e.g., Cutzu \& Edelman, 1994; Diwadkar \& McNamara, 1997). In the absence of compelling evidence to the contrary, we make the conservative assumption that a local system of reference is necessary to produce this generalization effect. 
We can only conclude that three views are not enough to exhibit view-invariant performance. We appreciate, of course, that it may be impossible, in the limit, to distinguish between a view-invariant theory and a multiple-representations theory: With a sufficiently large number of representations in memory, performance may appear to be view-invariant even though it is based on view-specific information (e.g., Tarr \& Pinker, 1989). Even so, our results suggest that the orientation-independent performance observed in previous studies (e.g., Hintzman et al., 1981; Presson et al., 1987) is probably attributable to the existence of multiple representations in memory rather than to the development of an orientation-independent representation of the space.

As acknowledged in the introduction, this interpretation of our data rests on the assumption that angular error in judgments of relative direction is an index of the extent to which spatial relations were inferred, with higher levels of error corresponding to greater levels of mental inference and computation. Two lines of evidence are broadly consistent with this assumption. First, the effects reported in angular error were replicated in response latency, suggesting that extra mental computation was needed to make judgments of relative direction from unfamiliar headings. Second, when participants were asked to describe the strategies they used to make judgments of relative direction, $91 \%$ reported that they started by retrieving a study view and then mentally transformed it to determine what the layout would look like from the novel view. A very small number of participants (1\%) reported a triangulation strategy whereby they essentially retrieved vectors defined by the various locations and computed the angle between them. The remaining $8 \%$ of the participants were unable to articulate the strategies they used. These observations are consistent with our assumption that the difference in accuracy between familiar and unfamiliar headings is caused by a difference in the extent to which spatial relations must be inferred.

A subtle but important property of our results is that participants interpreted the layouts in terms of spatial reference systems that were, in an important sense, unidirectional: There was rarely savings in angular error for headings that differed by $180^{\circ}$ from familiar headings (but for an example of such an effect, see Fig. 11), and with the notable exception of Experiments 2 and 4, there was little savings for headings orthogonal to familiar headings. Put another way, establishing "north" did not necessarily entail establishing "'south," "east,", or "west." Our findings parallel results on form perception. For example, the act of interpreting an ambiguous figure by designating a particular part or region as the top does not confer the ability to easily recognize the figure when it is upside down. It is natural to think of directional terms as existing in pairs; if a figure has a top, it must have a bottom; north implies south, and so on. Apparently these dualities may not exist in spatial perception and memory.

Is this pattern of results, and perhaps even the general pattern of orientation dependence, caused by the absence of locomotion? We did not allow partici- 
pants to walk around in the rooms because we wanted to have some measure of control over the number and the types of views they experienced. However, people typically move around in an environment, either under their own power or in a vehicle of some kind, when learning it for the first time. Does locomotion matter? Preliminary studies in our laboratory indicate that it does not. For example, in the learning phase of one experiment, participants studied the layout from a fixed vantage point, and then, after closing their eyes for a brief period of time, were asked to place all of the objects on their correct locations. No restrictions were placed on participants' movements. Angular error in judgments of relative direction evinced a sawtooth pattern, nearly identical to that of the aligned $\left(0^{\circ}\right)$ group of Experiment 2 (see Fig. 5).

It is important to recognize that, according to the theory we are proposing, the dominance of egocentrically defined spatial reference systems arises from the relative lack of salient, alternative, environmental reference systems. Environments rarely have directions as salient as those defined by experience. We hypothesize, however, that it may be possible to design environments or layouts in which external reference systems dominate egocentric reference systems. Imagine, for example, learning a layout on a steep incline. Here the environment establishes a conceptual "north" (either up or down the hill) that may supercede any directions established by personal experience. Likewise, it may be possible to design a layout in which the intrinsic reference system defined by the collection of objects provides a salient direction that also supercedes egocentric experience. Ongoing experiments in our laboratory are investigating situations like these.

Although these experiments have raised many important questions, they have also provided at least partial answers to the questions posed in the introduction. According to the theoretical framework we have proposed, learning the spatial structure of a novel environment involves interpreting the layout in terms of a spatial reference system. This process is analogous to determining the "top" of a figure (e.g., Rock, 1973); in effect, conceptual " "north" is assigned to the layout, creating privileged directions in the environment. The system of reference for this interpretation is established primarily by egocentric experience; however, egocentric perspectives that are aligned with salient external reference systems, such as the axes and planes defined by the walls, the floor, the ceiling, roads, and so forth, are preferred to those that are not aligned. From this perspective, many classical distinctions between spatial reference systems, such as egocentric vs allocentric, are false dichotomies. Spatial memories may be allocentric in the sense that the spatial relationships within the environment are coded with respect to a reference system imposed by the perceived structure of the environment, but also egocentric in the sense that these environmental reference systems are defined by egocentric experience. Future work may shed light on how these and other systems of spatial reference provide us with the information we need to find our way in the world. 


\section{REFERENCES}

Acredolo, L. P. (1978). Development of spatial orientation in infancy. Developmental Psychology, 14, 224-234.

Acredolo, L. P. (1979). Laboratory versus home: The effect of environment on the nine-monthold infant's choice of spatial reference system. Developmental Psychology, 15, 666-667.

Acredolo, L. P., \& Evans, D. (1980). Developmental changes in the effects of landmarks on infant spatial behavior. Developmental Psychology, 16, 312-318.

Attneave, F., \& Reid, K. W. (1968). Voluntary control of frame of reference and slope equivalence under head rotation. Journal of Experimental Psychology, 78, 153-159.

Biederman, I., \& Gerhardstein, P. C. (1993). Recognizing depth-rotated objects: Evidence and conditions for three-dimensional viewpoint invariance. Journal of Experimental Psychology: Human Perception and Performance, 19, 1162-1182.

Carlson-Radvansky, L. A., \& Irwin, D. E. (1993). Frames of reference in vision and language: Where is above? Cognition, 46, 223-244.

Cutzu, F., \& Edelman, S. (1994). Canonical views in object representation and recognition. Vision Research, 34, 3037-3056.

Diwadkar, V. A., \& McNamara, T. P. (1997). Viewpoint dependence in scene recognition. Psychological Science, 8, 302-307.

Easton, R. D., \& Sholl, M. J. (1995). Object-array structure, frames of reference, and retrieval of spatial knowledge. Journal of Experimental Psychology: Learning, Memory, and Cognition, 21, 483-500.

Friedman, A., \& Hall, D. L. (1996). The importance of being upright: Use of environmental and viewer-centered reference frames in shape discriminations of novel three-dimensional objects. Memory \& Cognition, 24, 285-295.

Goldmeier, E. (1937). Über Ähnlichkeit bei gesehenen Figuren. Psychologische Forschung, 21, 146-209. [Translation (by Goldmeier): Similarity in visually perceived forms. Psychological Issues, 8, 1-135.]

Hermer, L., \& Spelke, E. S. (1994). A geometric process for spatial reorientation in young children. Nature, 370, 57-59.

Hermer, L., \& Spelke, E. (1996). Modularity and development: The case of spatial reorientation. Cognition, 61, 195-232.

Hinton, G. (1979). Some demonstrations of the effects of structural descriptions in mental imagery. Cognitive Science, 3, 231-250.

Hintzman, D. L., O’Dell, C. S., \& Arndt, D. R. (1981). Orientation in cognitive maps. Cognitive Psychology, 13, 149-206.

Köhler, W. (1940). Dynamics in psychology. New York: Liveright.

Learmonth, A. E., Newcombe, N. S., \& Huttenlocher, J. (1998, November). Disoriented children use landmarks as well as geometry to reorient. Paper presented at the 39th Annual Meeting of the Psychonomic Society, Dallas, TX.

Levinson, S. C. (1996). Frames of reference and Molyneux's question: Crosslinguistic evidence. In P. Bloom, M. A. Peterson, L. Nadel, \& M. F. Garrett (Eds.), Language and space (pp. 109-169). Cambridge, MA: MIT Press.

Logan, G. D. (1995). Linguistic and conceptual control of visual spatial attention. Cognitive Psychology, 28, 103-174.

Marr, D. (1982). Vision. San Francisco: Freeman.

McMullen, P. A., \&, Jolicoeur, P. (1990). The spatial frame of reference in object naming and discrimination of left-right reflections. Memory \& Cognition, 18, 99-115. 
Palmer, S. E. (1989). Reference frames in the perception of shape and orientation. In B. E. Shepp \& S. Ballesteros (Eds.), Object perception: Structure and process (pp. 121-163). Hillsdale, NJ: Erlbaum.

Pani, J. R., \& Dupree, D. (1994). Spatial reference systems in the comprehension of rotational motion. Perception, 23, 929-946.

Pani, J. R., Williams, C. T., \& Shippey, G. T. (1995). Determinants of the perception of rotational motion: Orientation of the motion to the object and to the environment. Journal of Experimental Psychology: Human Perception and Performance, 21, 1441-1456.

Presson, C. C., DeLange, N., \& Hazelrigg, M. D. (1987). Orientation-specificity in kinesthetic spatial learning: The role of multiple orientations. Memory \& Cognition, 15, 225-229.

Presson, C. C., \& Montello, D. R. (1994). Updating after rotational and translational body movements: Coordinate structure of perspective space. Perception, 23, 1447-1455.

Rieser, J. J. (1989). Access to knowledge of spatial structure at novel points of observation. Journal of Experimental Psychology: Learning, Memory, and Cognition, 15, 1157-1165.

Rieser, J. J., Guth, D. A., \& Hill, E. W. (1986). Sensitivity to perspective structure while walking without vision. Perception, 15, 173-188.

Rock, I. (1956). The orientation of forms on the retina and in the environment. American Journal of Psychology, 69, 513-528.

Rock, I. (1973). Orientation and form. New York: Academic Press.

Rock, I. (1992). Comment on Asch and Witkin's ' Studies in Space Orientation II.' Journal of Experimental Psychology: General, 4, 404-406.

Rock, I., \& Heimer, W. (1957). The effect of retinal and phenomenal orientation on the perception of form. American Journal of Psychology, 70, 493-511.

Roskos-Ewoldsen, B., McNamara, T. P., Shelton, A. L., \& Carr, W. S. (1998). Mental representations of large and small spatial layouts are orientation dependent. Journal of Experimental Psychology: Learning, Memory, \& Cognition, 24, 215-226.

Shelton, A. L., \& McNamara, T. P. (1997a). Multiple views of spatial memory. Psychonomic Bulletin \& Review, 4, 102-106.

Shelton, A. L., \& McNamara, T. P. (1997b). Representing space: reference frames and multiple views. In M. G. Shafto \& P. Langley (Eds.), Proceedings of the Nineteenth Annual Convention of the Cognitive Science Society (p. 1048). Mahwah, NJ: Erlbaum.

Sholl, M. J., \& Nolin, T. L. (1997). Orientation specificity in representations of place. Journal of Experimental Psychology: Learning, Memory, and Cognition, 23, 1494-1507.

Talmy, L. (1983). How language structures space. In H. L. Pick, Jr. \& L. P. Acredolo (Eds.), Spatial orientation: Theory, research, and application (pp. 225-282). New York: Plenum Press.

Tarr, M. J., \& Pinker, S. (1989). Mental rotation and orientation-dependence in shape recognition. Cognitive Psychology, 21, 233-282.

Tversky, B. (1981). Distortions in memory for maps. Cognitive Psychology, 13, 407-433.

Vetter, T., Poggio, T., \& Bülthoff, H. H. (1994). The importance of symmetry and virtual views in three-dimensional object recognition. Current Biology, 4, 18-23.

(Accepted February 25, 2001; published online July 5, 2001) 